\title{
Distal Chromosome 17q Loss in Barrett's Esophageal and Gastric Cardia Adenocarcinomas: Implications for Tumorigenesis
}

\author{
Elizabeth M. Petty, ${ }^{1 *}$ Linda M. Kalikin, ${ }^{1}$ Mark B. Orringer, ${ }^{2}$ and David G. Beer ${ }^{2}$ \\ ${ }^{1}$ Departments of Internal Medicine and Human Genetics, University of Michigan Medical Center, Ann Arbor, Michigan \\ ${ }^{2}$ Department of Surgery, University of Michigan Medical Center, Ann Arbor, Michigan
}

The molecular genetic mechanisms underlying esophageal cancer are poorly understood. However, a novel gene that may be involved in esophageal carcinogenesis was recently localized by others to distal $17 q$ by linkage analysis of kindreds with palmoplantar keratoderma and squamous cell carcinoma of the esophagus. To help determine whether a distal 17q gene may also be involved in the pathogenesis of primary Barrett's esophageal and gastric cardia adenocarcinomas, we performed loss of heterozygosity (LOH) analysis of 21 Barrett's and 18 gastric cardia adenocarcinomas at loci spanning 17q: cen-BRCA1-SSTR2-D17S2058D175929-D17S722-D175937-D17S802-tel. Over 50\% of the Barrett's and cardia adenocarcinomas demonstrated loss of an allele at one or more informative distal 17q markers. One common overlapping region of loss involved loci mapped to distal 17q24-proximal 17q25, which tentatively defines a potential chromosomal region distal to $B R C A 1$ involved in the pathogenesis or progression of both types of adenocarcinomas. $\mathrm{LOH}$ analysis of DNA from matched microdissected sections of Barrett's metaplasia suggested that loss of D17S2058 in this region may be an early event in the malignant transformation of Barrett's metaplasia. No statistically significant correlations between $17 q \mathrm{LOH}$ and tumor stage or patient survival were noted. In summary, LOH mapping of 17q in Barrett's and cardia adenocarcinomas suggests the existence of at least one putative distal $17 q$ tumor suppressor gene involved in the pathogenesis of these tumors. Mol. Carcinog. 22:222-228, 1998. () 1998 Wiley-Liss, Inc.

Key words: tumor suppressor gene; loss of heterozygosity; malignancy; cancer; gene mapping

\section{INTRODUCTION}

The incidence and mortality rates of esophageal adenocarcinoma continue to rise for men in the United States, in contrast to the decreasing incidence reported for many other types of solid tumor malignancies $[1,2]$. Unfortunately, relatively little is known about the multistep molecular mechanisms underlying this disease as compared with those of other solid tumor malignancies. It has been demonstrated that esophageal adenocarcinomas often arise from Barrett's columnar metaplasia of the esophagus, which is thought to be acquired secondary to chronic gastroesophageal reflux $[3,4]$. It is estimated that approximately $10 \%$ of individuals with Barrett's esophageal metaplasia may undergo malignant transformation of the esophagus [3]. At present, many of the critical genetic and nongenetic factors underlying this malignant transformation remain unclear. We hope that improved understanding of the molecular genetic events predisposing individuals to increased susceptibility for malignant transformation will facilitate improved diagnostic testing and prognostic management for affected individuals and individuals at risk. Pathological and immunohistochemical analyses with p53 and intestinal enzyme expression suggested that molecular changes consis- tent with Barrett's adenocarcinoma are also present in adenocarcinomas of the gastric cardia (gastroesophageal junction) [5-7], suggesting that similar genetic mechanisms may underlie the development of both tumor types.

Several genes are hypothesized to play a role in the progression of esophageal carcinogenesis (including the TP53, CDKN2, and APC genes) based on genetic studies, but it is quite likely that additional as-yet-unidentified genes may also be involved [819]. The development of esophageal and gastric cardia tumors is thought to reflect a clonal multistep process involving the accumulation of mutations in growth regulatory genes, similar to the multistep process of tumorigenesis so elegantly described for colorectal cancer [20]. Linkage analysis of kindreds with palmoplantar keratoderma and esophageal squamous cell carcinoma localized a novel gene for

\footnotetext{
* Correspondence to: Division of Molecular Medicine and Genetics, Department of Internal Medicine, University of Michigan Medical Center, 4301 MSRB III, Box 0638, Ann Arbor, Ml 48109-0638.

Received 12 August 1997; Revised 20 January 1998; Accepted 24 February 1998

Abbreviations: LOH, loss of heterozygosity; PCR, polymerase chain reaction.
} 
this syndrome to 17q24-25 [21-24]. We have also mapped a putative tumor suppressor gene or genes involved in breast and ovarian cancer distal to $17 \mathrm{q} 25$ by loss of heterozygosity (LOH) studies $[25,26]$. LOH studies, most often in concert with other associated positional cloning strategies, have provided critical localization information for the cloning of several tumor suppressor genes [27].

To date, there have been few detailed LOH studies of distal $17 \mathrm{q}$ in esophageal and gastric cardia neoplasms, and most have been limited to proximal 17q, where $B R C A 1$, the culprit gene for familial early-onset breast and ovarian cancer, resides. No detailed LOH studies of distal chromosome 17q have been published. We analyzed esophageal and gastric cardia tumors for $\mathrm{LOH}$ on $17 \mathrm{q}$ to help determine the potential roles, if any, of putative distal $17 \mathrm{q}$ tumor suppressor genes in the pathogenesis of these tumors and to determine if the same $17 \mathrm{q} 24-25$ genetic region demonstrating linkage to squamous cell carcinoma of the esophagus associated with palmoplantar keratoderma demonstrates LOH in sporadic esophageal and cardia adenocarcinomas. We also wanted to determine whether the discrete region of $17 \mathrm{q} 25$ $\mathrm{LOH}$ we previously detected in breast and ovarian neoplasms is also lost in esophageal and gastric cardia tumors, which would suggest localization of a novel tumor suppressor gene involved in multiple solid tumors and akin to some of the cell-cycle regulators like p16 [28].

\section{MATERIALS AND METHODS}

\section{Samples}

DNA samples from 21 Barrett's esophageal adenocarcinomas and 18 gastric cardia tumors were extracted from dissections of frozen tissue obtained at the time of esophagotomy at the University of Michigan Medical Center. Each patient's age at diagnosis, sex, tumor type, TNM stage, and months of survival (based on last follow-up examination) after diagnosis were recorded. Careful pathological analysis of tumor margins on $5-\mu \mathrm{m}$ cryostat sections was conducted before careful dissection of frozen tumor tissue specimens to enrich for the targeted tumor tissue.
DNA was extracted by standard methods as previously described [15]. The tumor cell nuclei content of each sample, estimated by microscopic analysis of tumor specimens and margins, exceeded $80 \%$. Samples demonstrating more than 20\% stromal and normal inflammatory cells were not used for $\mathrm{LOH}$ analysis to avoid uninterpretable results due to significant admixture of normal cells in tumor samples. Matched normal DNA samples were extracted from normal esophageal or gastric tissue by using standard techniques previously reported [15]. For 11 of the Barrett's adenocarcinomas, matched DNA samples were also prepared from minute discrete regions of Barrett's metaplastic epithelium. These specimens were microdissected from normal tissue on $5-\mu \mathrm{m}$ cryostat sections under a dissecting microscope to enrich for metaplastic cells by using previously reported methods [26]. Using this method of microdissection, we were able to obtain samples in which well over 90\% of the nuclei were from Barrett's metaplastic cells.

\section{LOH Analysis}

Polymerase chain reactions (PCRs) of matched tumor and normal pairs were conducted with eight chromosome 17q polymorphic markers [29] (Table 1) in which one primer was end-labeled with T4 polynucleotide kinase and $\left[\gamma^{32} \mathrm{P}\right] \mathrm{dATP}$. The PCR annealing conditions used were based on optimized conditions for LOH previously reported [26]. Samples were electrophoresed at $70 \mathrm{~W}$ on $5 \%$ denaturing formamide-containing polyacrylamide gels and exposed to $\mathrm{x}$-ray film from $0.5-72 \mathrm{~h}$ to generate light and dark exposures for all gels. LOH was visually scored by two independent observers when an allele was absent or clearly displayed a markedly decreased signal intensity in tumor tissue. Tumors that visually exhibited a reduction in signal intensity but not complete absence were also subjected to digital densitometry analysis (Alpha-Inotech Corporation) to calculate allelic imbalance ratios to confirm our visual inspection. When over $50 \%$ allelic imbalance was detected in tumor alleles compared with normal tissue alleles, the tumor was characterized as

Table 1. 17q Loci Analyzed in Esophageal and Gastric Cardia Tumors

\begin{tabular}{llcc}
\hline Locus & Primers* & Heterozygosity frequency $^{\dagger}$ & Map location $^{\ddagger}$ \\
\hline BRCA1 & AFM248yg9 & 0.80 & $17 \mathrm{q} 21$ \\
BRCA1 & S975 & 0.65 & $17 \mathrm{q} 21$ \\
SSTR2 & SSTR2 1/5 & 0.88 & $17 \mathrm{q} 24$ \\
D17S2058 & CTT16 & 0.78 & $17 \mathrm{q} 24-25$ \\
D17S929 & AFM074zf7 & 0.60 & $17 \mathrm{q} 24-25$ \\
D17S722 & nm00060up & 0.84 & $17 \mathrm{q} 25$ \\
D17S937 & AFM107ye3 CAVGT & 0.72 & $17 \mathrm{q} 25$ \\
D17S802 & AFM210xa5 CAVGT & 0.82 & $17 \mathrm{q} 25$ \\
\hline
\end{tabular}

*Primer sequences and general amplification conditions are from the Online Genome Data Base [29] (http://gdbwww.gdb.org/).

${ }^{\dagger}$ Heterozygosity frequencies were calculated from our laboratory data set.

FLoci are listed from proximal to distal $17 q$ based on database reports $[29,30]$ and our physical mapping (unpublished data). 
having LOH. In all cases, densitometry analyses were concordant with visual inspection.

\section{RESULTS}

The LOH patterns for distal 17q loci in both Barrett's esophageal and gastric cardia adenocarcinomas were strikingly similar, as demonstrated in Figure $1 \mathrm{~A}$ and B. No LOH was seen in three squamous cell carcinomas analyzed in the same manner (data not shown). Most of the samples provided clearly interpretable $\mathrm{LOH}$ results, as shown in Figure 2. Most of the tumors had clear regions of allelic loss and regions of allelic retention, indicating that our dissected samples provided adequate enrichment of tumor tissue for LOH analysis (Figure 2). For tumor samples in which no $\mathrm{LOH}$ was present at any of the analyzed loci, it is difficult to say with absolute certainty whether admixture of normal cells in the tumor samples may have hampered detecting regions of $\mathrm{LOH}$. It is possible that the $\mathrm{LOH}$ rate may have been underestimated, because some samples may not have exhibited detectable LOH because of admixture of normal cells. However, given that visual inspection of cryostat sections of these tumors and estimation of their tumor nuclei content showed that these tumors were not different from tumors in which clear $\mathrm{LOH}$ was noted, we are confident that our results accurately represent the true LOH. In fact, only in a minority of informative tumor samples, less than $2 \%$, could we not determine with confidence whether or not allelic imbalance was present even after reamplification and reanalysis of samples. This was most often due to poor amplification of the tumor sample.

The overall rates of $\mathrm{LOH}$ for seven loci in all tumors studied are presented in Table 2 and varied from a low rate of four of 32 informative tumors (12.5\%) at BRCA1 (when data for both informative markers are combined) to the highest rates of seven of 20 informative tumors (35\%) at D17S929 and 12 of 36 informative tumors (33\%) at D17S2058 (Figure 2). Eleven Barrett's adenocarcinoma samples (numbers $8,10,18,22,26,28,30,32,83,89$, and 92) were also compared with available matched microdissected specimens representing potentially premalignant Barrett's metaplastic epithelium at loci where LOH was present in the tumor specimen. Concordant LOH was seen in the matched premalignant Barrett's epithelium and adenocarcinoma samples for tumor sample 10 at D17S2058 (Figure 3) and tumor sample 30 at D17S722 and tumor sample 92 at BRCA1 (data not shown). Interestingly, the matched premalignant Barrett's epithelium sample for adenocarcinoma sample 22 was concordant for LOH at D17S2058 but was discordant with the matched adenocarcinoma at informative flanking loci, as the tumor but not the premalignant Barrett's specimen demonstrated LOH at these loci.

Each patient's age, sex, tumor stage, and months of survival after diagnosis at last follow-up are also presented in Figure 1A and B. Most of the samples, $86 \%$ of the Barrett's adenocarcinomas and $89 \%$ of the cardia adenocarcinomas, were from male patients. It is interesting that the average age at diagnosis for men with Barrett's adenocarcinomas was $63.5 \mathrm{yr}$, whereas the average age of onset for the women with Barrett's adenocarcinomas was much higher (78 yr; not statistically significant). The average age at diagnosis of cardia tumors in men was similar to those diagnosed with Barrett's tumors (64 yr), but the two women with cardia tumors were diagnosed in their 50s, at a lower average age than women with Barrett's tumors. The mean survival time for individuals diagnosed with either tumor was approximately 9 mo. Most of the samples of both tumor types exhibited positive p53 nuclear accumulation (data not shown), which did not correlate with presence or absence or distal 17q loss. In fact, in this relatively small sample, there were no statistically significant correlations between or identifiable trends in $17 \mathrm{q} \mathrm{LOH}$ and patient survival, age, or sex or tumor stage.

\section{DISCUSSION}

Our LOH data suggest that one or more putative genes involved in Barrett's and gastric cardia adenocarcinomas may map to distal $17 \mathrm{q}$, as more than $50 \%$ of tumors studied demonstrated loss at one or more distal 17q loci. The most common region of loss was at $17 \mathrm{q} 24$-proximal $17 \mathrm{q} 25$, represented by loci D17S2058 and D17S929, suggesting that a critical gene may lie between these loci. This region of loss is clearly distinct from and distal to $B R C A 1$, at which only $12.5 \%$ of all the adenocarcinomas demonstrated loss. The fact that less than $25 \%$ loss was seen at $17 \mathrm{q}$ loci flanking the region of highest $\mathrm{LOH}$ supports the hypothesis that loss at D17S2058 and D17S929 probably does not simply reflect background loss. Whether this region is a region that is relatively unstable in the progression of malignant transformation of solid tumors rather than the location of a single putative gene cannot be determined from this study. However, the previously reported findings of LOH less than $10 \%$ at D17S2058 in other solid tumors, including lung, prostate, breast, and ovarian adenocarcinomas, suggests that this region is not a region highly susceptible to breakage in solid tumor progression [26].

The precise physical distance between these two distal 17q loci remains somewhat uncertain, given current information in genomic database reports, as the loci have not yet been physically mapped with respect to one another on the same currently available maps of the region. However, extrapolation of the loci's respective locations compared with those of well-mapped flanking loci suggests that they are probably approximately 3-4 cM apart, based on recent sex-averaged CEPH-Genethon linkage maps of 


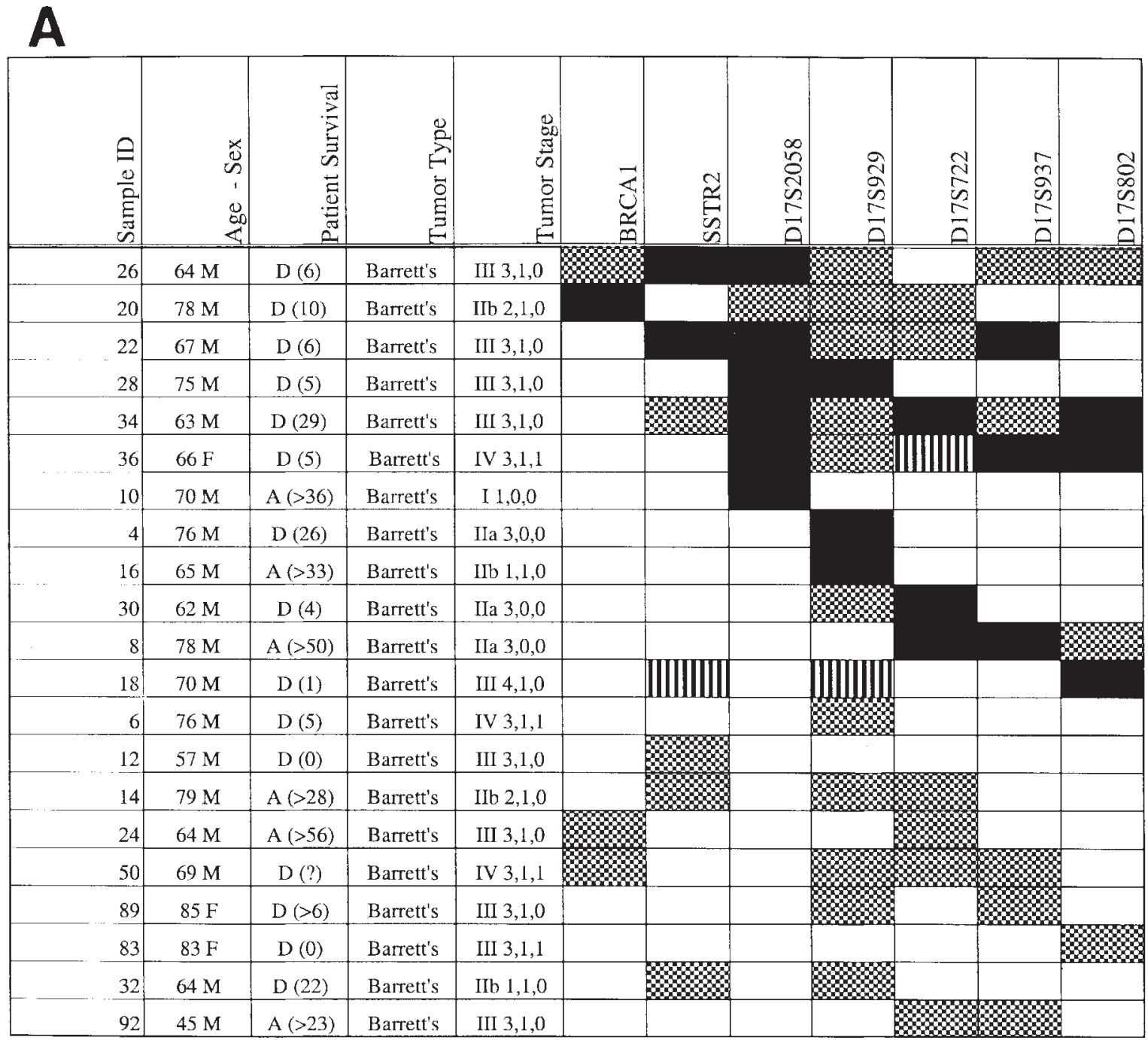

Figure 1. Summary of data for Barrett's adenocarcinoma samples (A) and gastric cardia adenocarcinomas (B). Each patient's age at diagnosis, sex, and months of survival after diagnosis ( $D=$ deceased, $A=$ alive) are shown when known. The TNM stage and type classified by the University of Michigan's Surgical Pathology Department are also presented.

the region [30]. Obviously, further development of physical and genetic maps in this region is needed to clarify the distances between these markers and confirm their orientation with respect to one another. Other scattered regions of $17 \mathrm{q}$ loss in these tumors may suggest that more than one $17 \mathrm{q}$ gene is involved or may simply reflect random chromosomal instability in tumor progression.

The highest rates of LOH (more than 30\%) were observed at D17S929 and D17S2058, the same loci defining the smallest common interval of loss. LOH at D17S2058 in two of four Barrett's metaplastic epithelium samples in which the matched Barrett's adenocarcinoma demonstrated LOH tentatively suggests that $\mathrm{LOH}$ in this region may be an early event in the pathogenesis of Barrett's adenocarci-
For $\mathrm{LOH}$, the loci are listed proximal to distal. Solid boxes indicate loss and open boxes retention of heterozygosity, checked boxes indicate noninformative results, and vertical bars indicate microsatellite instability. The data for the two intragenic $B R C A 1$ polymorphic markers are combined.

noma. This $\mathrm{LOH}$ in the metaplastic lesion is not as clean as in the tumor sample, suggesting that the tumor cells are a clonal population of cells, whereas the metaplastic cells are not (Figure 3). In one of the matched sets of normal, metaplastic, and tumor samples (tumor number 22), the adenocarcinoma demonstrated loss at three distal 17q loci (SSTR2, D17S2058, and D17S937), but the matched nondysplastic Barrett's epithelium demonstrated loss only at D17S2058, further supporting the hypothesis that alterations of a gene in this region may be an early event. In addition, a matched lung adenocarcinoma sample (thought to be a second primary tumor pathologically) from the patient whose esophageal sample is number 10 also demonstrated loss at D17S2058 (data not shown). This may imply that 


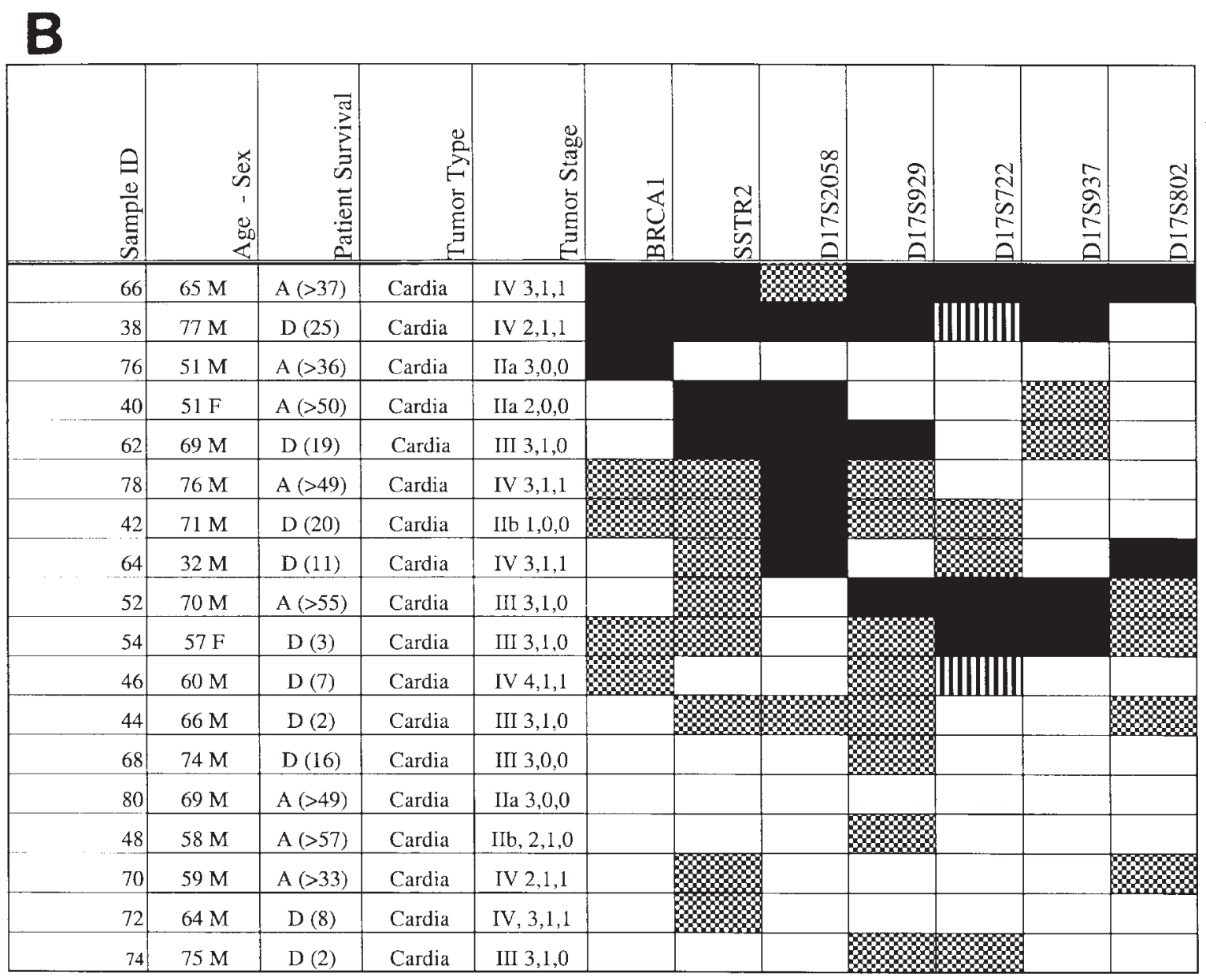

Figure 1. (continued).

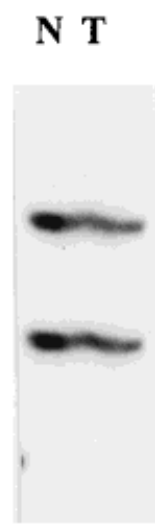

A.

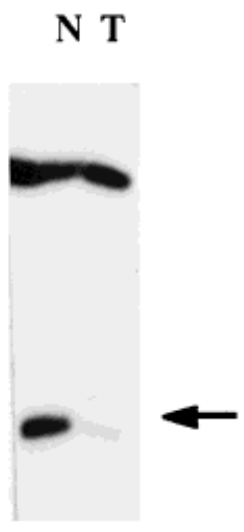

B.

\section{N T}

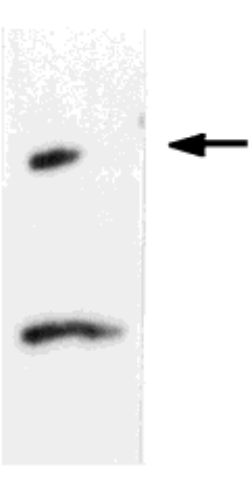

C.

Figure 2. Examples of LOH analysis at D1752058, the locus where the highest rate of loss was observed, in three different esophageal adenocarcinomas. $\mathrm{N}$, normal sample; $\mathrm{T}$, matched tumor sample. (A) No loss of heterozygosity as amplification of the larger and smaller alleles in the tumor sample produced bands of relatively equal intensities as analyzed by autoradiography after denaturing polyacrylamide gel electrophoresis of the amplified PCR product. (B) Loss of the smaller allele in this tumor is indicated by the arrow. (C) Loss of the larger allele in another tumor is noted by the arrow.

the lung tumor was actually a metastatic lesion rather than a primary second tumor in this individual or that the putative gene may also play a role in lung adenocarcinomas. We subsequently analyzed 19 adenocarcinomas of the lung for LOH of D17S2058 by using the same methods described above and did not find significant loss (less than 10\%) in the tumors we studied. This suggests that it is less likely that

Table 2. Overall Rates of Loss in Esophageal and Gastric Cardia Adenocarcinomas for 17q Loci

\begin{tabular}{lrl}
\hline & $\begin{array}{c}\text { No. of tumors with loss/ } \\
\text { no. of informative tumors }\end{array}$ \\
\hline Locus & $4 / 32$ & $(12.5 \%)$ \\
BRCA1* & $6 / 27$ & $(22 \%)$ \\
SSTR2 & $12 / 36$ & $(33 \%)$ \\
D17S2058 & $7 / 20$ & $(35 \%)$ \\
D175929 & $5 / 31$ & $(16 \%)$ \\
D17S722 & $7 / 32$ & $(22 \%)$ \\
D175937 & $5 / 32$ & $(16 \%)$ \\
D17S802 & &
\end{tabular}

* $\mathrm{LOH}$ for this locus reflects data combining BRCA1 polymorphic markers. 


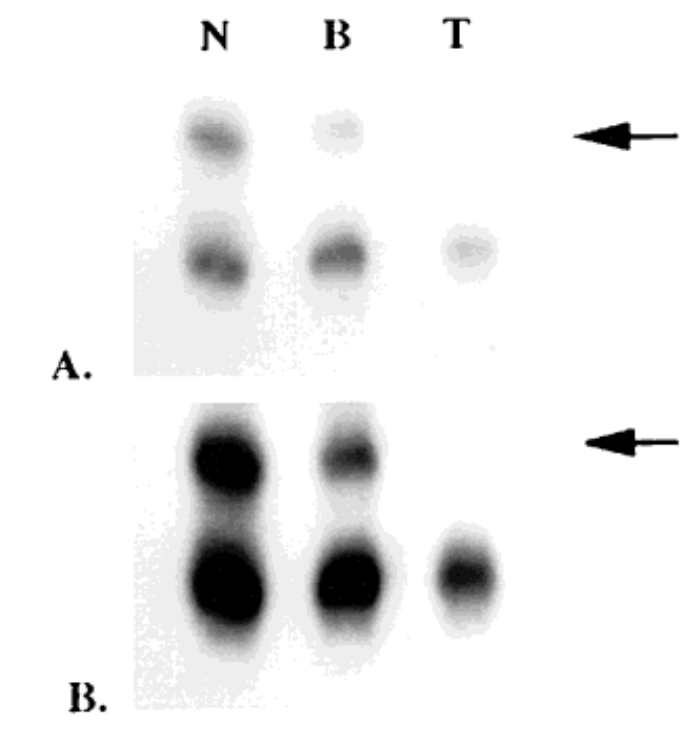

Figure 3. Light (A) and dark (B) autoradiograph exposures of LOH at D1752058 in esophageal adenocarcinoma sample number 10 . This tumor demonstrated loss only at D1752058. Clean loss of the larger allele (arrow) is demonstrated in the tumor sample $(\mathrm{T})$ when compared with the matched normal sample (N). The matched metaplastic Barrett's sample (B) demonstrates decreased intensity of the same larger allele that is absent in the tumor sample (T), suggesting that some of the cells from the Barrett's metaplasia sample (B) had lost this allele but that it is not a clonal population of cells.

$\mathrm{LOH}$ in this region is a common event in primary lung adenocarcinomas (unpublished data). Similarly, prostate adenocarcinomas do not demonstrate $17 \mathrm{q} 25$ loss [26]. Thus, $\mathrm{LOH}$ in this distal 17q24-proximal $17 q 25$ region is not observed in adenocarcinomas of different tissue types. This supports our hypotheses that a specific tumor suppressor gene or genes map to this particular distal $17 \mathrm{q}$ region and that the $\mathrm{LOH}$ we observed in esophageal and gastric cardia tumors probably does not simply reflect random distal $17 \mathrm{q}$ chromosomal instability associated with generalized tumor progression. Clearly, continued analysis of adenocarcinomas for involvement of this putative distal 17q gene or genes in relationship to involvement of other putative cancer genes is needed to elucidate the hypothesized multistep processes that are associated with esophageal carcinogenesis. The most common region of loss in the adenocarcinomas is clearly proximal to the specific $17 \mathrm{q} 25$ region of $\mathrm{LOH}$ previously identified in breast and ovarian tumors [26], suggesting that different distal $17 \mathrm{q}$ genes are probably involved in these different solid tumors.

The patterns of 17q LOH in Barrett's and gastric cardia adenocarcinomas were strikingly similar, supporting the hypothesis that the tumors have similar molecular mechanisms of development, as previously proposed [7]. No significant statistical correlations could be drawn between distal 17q LOH and other clinicopathological data. Only one of the tumors analyzed demonstrated loss at all informative $17 \mathrm{q}$ loci, and it was a stage IV gastric cardia adenocarcinoma that also demonstrated positive p53 protein nuclear immunoreactivity. Interestingly, five of nine other stage IV tumors analyzed showed no loss of $17 q$ alleles. That most of the samples were from men simply reflects the observed population incidence of these types of esophageal and gastric cardia cancer.

The $17 \mathrm{q}$ LOH we observed is within the same general region where others have mapped a gene involved in squamous cell carcinoma of the esophagus associated with autosomal dominant palmoplantar keratoderma [21-24]. Our region of LOH may be slightly proximal to the locus they mapped by linkage analysis. This may reflect the relatively poor physical mapping of the region to date, may suggest that two different genes are involved, or may demonstrate a specific area of $\mathrm{LOH}$ related to a nearby culprit gene. Although LOH mapping has clearly facilitated the ultimate isolation of several putative tumor suppressor genes [27], gene localization by LOH mapping has not always been precisely consistent with linkage analysis localization of a gene within the same 3-4 cM interval, as recently reported in the mapping and cloning of the patched gene causing basal cell carcinomas [31]. This may reflect some limitations in the methodology of accurately assessing $\mathrm{LOH}$ or may suggest that the LOH may have occurred in some upstream regulatory region of the putative gene, altering its expression or function. We found no $17 \mathrm{q} \mathrm{LOH}$ in three squamous cell carcinomas of the esophagus, which was unexpected (unpublished data). Obviously, analysis of additional samples is required to support or refute the hypothesis that a tumor suppressor gene involved in sporadic primary esophageal squamous cell carcinomas maps to this region of $17 \mathrm{q}$. However, it is very intriguing that the region implicated by linkage analysis is within the same region where we noted the most common overlapping of loss as well as the highest rate of $17 \mathrm{q} \mathrm{LOH}$. This may suggest that in the gene in which germline mutations predispose individuals to development of an autosomal dominant syndrome consisting of esophageal cancer associated with palmoplantar keratoderma, somatic mutations predispose individuals to sporadic types of esophageal cancer. The association of cancer predisposition with syndromes characterized by abnormal nonmalignant phenotypes has been well described. An example is Gorlin's syndrome, which is characterized by a variety of developmental defects and multiple basal cell carcinomas. Affected patients have germline mutations in the patched gene. Sporadic basal cell carcinomas are also associated with somatic mutations in the patched gene [31].

In summary, $\mathrm{LOH}$ analysis of distal $17 \mathrm{q}$ loci in Barrett's esophageal and gastric cardia adenocarcinomas suggested that genetic alterations of distal 17q may be involved in the pathogenesis of these tumors. The smallest common interval of loss and 
highest rates of loss at D17S929-D17S2058, along with the early loss detected at D17S2058 in Barrett's metaplasia samples, suggested that a putative tumor suppressor gene may be located near these distal 17q24-proximal 17q25 loci. To determine the significance of distal $17 \mathrm{q}$ genetic alterations in the pathogenesis of esophageal and gastric cardia adenocarcinoma, further efforts are under way to better localize and isolate this putative gene and analyze its role in esophageal cancer.

\section{ACKNOWLEDGMENTS}

We thank Mr. I. N. Odocha for his technical assistance and Ms. Maggie VanOvermeer for assistance in manuscript preparation. This work was supported in part by NIH-NCI KO8 CA 66613 (to EMP) and University of Michigan Cancer Center Funds (to EMP and DGB).

\section{REFERENCES}

1. American Cancer Society. Cancer Facts and Figures. American Cancer Society, Atlanta, GA, 1994.

2. Blot WJ, Devesa SS, Kneller RW, Fraumeni JF. Rising incidence of adenocarcinoma of the esophagus and gastric cardia. JAMA 265:1287-1289, 1991.

3. Fennerty MB, Barrett's esophageus-cancer risk, biology and therapeutic management. Aliment Pharmacol Ther 7:339-345, 1993.

4. Spechler SJ, Goyal RK. Barrett's esophagus. N Engl J Med 315:362-371, 1986.

5. Wu GD, Beer DG, Moore JH, Orringer MB, Appelman HD, Traber PG. Sucrase-isomaltase gene expression in Barrett's esophagus and adenocarcinoma. Gastroenterology 105:837-844, 1993.

6. Moore JH, Lesser EJ, Erdody DH, Natale RB, Orringer MB, Beer DG. Intestinal differentiation and p53 gene alterations in Barrett's esophagus and esophageal adenocarcinoma. Int J Cancer 56:487-493, 1994.

7. Iannettoni MD, Lee SS, Bonnell MR, et al. Detection of Barrett's adenocarcinoma of the gastric cardia with sucrase isomaltase and p53. Ann Thorac Surg 62:1460-1465, 1996.

8. Mori T, Aoki T, Matsubara T, et al. Frequent loss of heterozygosity in the region including BRCA1 on chromosome $17 \mathrm{q}$ in squamous cell carcinomas of the esophagus. Cancer Res 54: 1638-1640, 1994

9. Krishnadath KK, Tilanus HW, van Blankenstein M, et al. Accumulation of genetic abnormalities during neoplastic progression in Barrett's esophagus. Cancer Res 55:1971-1976, 1995

10. Hunter S, Gramlich T, Abbott K, Varma V. Y chromosome loss in esophageal carcinoma: An in situ hybridization study. Genes Chromosomes Cancer 8:172-177, 1993.

11. Mori T, Yanagisawa $A$, Kato $Y$, et al. Accumulation of genetic alterations during esophageal carcinogenesis. Human Mol Genet 3:1969-1971, 1994a.

12. Zhuang Z, Vortmeyer AO, Mark EJ, et al. Barrett's esophagus: Metaplastic cells with loss of heterozygosity at the APC gene locus are clonal precursors to invasive adenocarcinoma. Cancer Res 56:1961-1964, 1996.

13. Barrett MT, Scutte M, Kern SE, Reid BJ. Allelic loss and mutational analysis of the DPC 4 gene in esophageal adenocarcinoma. Cancer Res 56:4351-4353, 1996.

14. Boynton RF, Blount PL, Yin J, et al. Loss of heterozygosity involving the APC and MCC genetic loci occurs in the majority of human esophageal cancers. Proc Natl Acad Sci USA 89:3385-3388, 1992.

15. al-Kasspooles MF, Moore JH, Orringer MB, Beer DG. Amplification and overexpression of the EGFR and erbB2 genes in human esophageal adenocarcinomas. Int J Cancer 54:213-219, 1993.

16. Whang-Peng J, Banks-Schlegel SP, Lee EC. Cytogenetic studies of esophageal carcinoma cell lines. Cancer Genet Cytogenet 45:101-120, 1990

17. Bongiorno PF, al-Kasspooles M, Lee SW, et al. E-cadherin expression in primary and metastatic thoracic neoplasms and in Barrett's esophagus. Br J Cancer 71:166-172, 1995.

18. Aoki T, Mori T, Du X, Nisihira T, Matsubara T, Nakamura Y. Allotype study of esophageal carcinoma. Genes Chromosomes Cancer 10:177-182, 1994.

19. Swift A, Risk JM, Kingsnorth AN, Wright TA, Myskow M, Field JK. Frequent loss of heterozygosity on chromosome 17 at 17q11.2-q12 in Barrett's adenocarcinoma. Br J Cancer 71(5):995998, 1995

20. Fearon ER, Vogelstein B. A genetic model for colorectal tumorigenesis. Cell 61:759-767, 1990.

21. Risk JM, Field EA, Field JK, et al. Tylosis esophageal cancer mapped. Letter. Nat Genet 8:319-320, 1994.

22. Hennies H-C, Hagedorn M, Reis A. Palmoplantar keratoderma in association with carcinoma of the esophagus maps to chromosome $17 q$ distal to the keratin gene cluster. Genomics 29:537540, 1995.

23. Kelsell DP, Risk JM, Leigh IM, et al. Close mapping of the focal non-epidermolytic palmoplantar keratoderma (PPK) locus associated with esophageal cancer (TOC). Hum Mol Genet 5:857860, 1996.

24. Stevens HP, Kelsell DP, Bryant SP, et al. Linkage of an American pedigree with palmoplantar keratoderma and malignancy (palmoplantar ectodermal dysplasia type III) to 17q24. Arch Dermatol 132(6):640-651, 1996.

25. Kalikin LM, Qu X, Frank TS, et al. Fine tumor deletion mapping defines the location of a putative $17 q 25$ gene involved in breast carcinogenesis. Genes Chromosomes Cancer 17:64-68, 1996.

26. Kalikin LM, Odocha IM, Svoboda SM, et al. Breast and ovarian tumors, but not prostate tumors, demonstrate coincident region of distal 17q loss of heterozygosity. Oncogene 14:1991-1994, 1997.

27. Knudson AG. Antioncogenes and human cancer. Proc Natl Acad Sci USA 90:10914-10921, 1993.

28. Kamb A, Gruis NA, Weaver-Feldhaus J, et al. A cell cycle regulator potentially involved in genesis of many tumor types. Science 264:436-440, 1994.

29. Fasman KH, Cuticchia AJ, Kingsbury DT. The GDB Human Genome Data Base, 1994. Nucleic Acids Res 17:3462-3469, 1994.

30. Dib C, Faure S, Fizames $C$, et al. A comprehensive map of the human genome based on 5,264 microsatellites. Nature 380:152154, 1996.

31. Hahn H, Wicking C, Zaphiropoulous PG, et al. Mutations of the human homologue of Drosophila patched in the nevoid basal cell carcinoma syndrome. Cell 85:841-851, 1996. 